\title{
Pharmacokinetic Interaction of Rifampicin with Oral Versus Intravenous Anticancer Drugs: Challenges, Dilemmas and Paradoxical Effects Due to Multiple Mechanisms
}

\author{
Nuggehally R. Srinivas ${ }^{1}$
}

Published online: 20 April 2016

(C) The Author(s) 2016. This article is published with open access at Springerlink.com

\begin{abstract}
Since many drugs are cytochrome P450 (CYP)3A4 substrates, it has become common practice to assess drug-drug interaction (DDI) potential with a CYP3A4 inhibitor (ketoconazole) or inducer (rifampicin) in early drug development. Such an evaluation is relevant to anticancer drugs with metabolism governed by CYP3A4. DDIs with rifampicin are complex, involving other physiological mechanisms that may impact overall pharmacokinetics. Our objective was to study and delineate such mechanisms for oral versus intravenous anticancer drugs. We hypothesized that DDIs between anticancer drugs and rifampicin were primarily driven by CYP3A4 induction. This hypothesis was proven for the oral anticancer drugs; however, in some cases, other intrinsic mechanisms such as P-glycoprotein (Pgp)/UDP glucuronosyl transferase (UGT) induction and transporter inhibition may have played an important role alongside the induced CYP3A4 enzymes. The hypothesis that CYP3A4 induction would decrease drug exposure appeared paradoxical for intravenous romidepsin and-to a somewhat lesser extent-for cabazitaxel. In light of this dilemma in the interpretation of the pharmacokinetic data with rifampicin, several questions require further consideration. Given the complexity and paradoxical effects arising with DDIs with rifampicin, the continued preference for rifampicin as CYP3A4 inducer needs immediate re-appraisal.
\end{abstract}

Nuggehally R. Srinivas

srini.suramus@yahoo.com

1 Suramus Bio, Drug Development, 29th Main, 10th Cross, JP Nagar I Phase, Bangalore 560078, Karnataka, India

\section{Key Points}

Rifampicin is the preferred probe to facilitate cytochrome P450 (CYP)-3A4 induction in drugdrug interaction studies involving drugs metabolized via the CYP3A4 enzyme.

Since rifampicin can affect other physiological processes besides CYP3A4 induction, it may lead to paradoxical observations as illustrated by the data gathered for oral versus intravenous anticancer drugs.

In light of the complexity and challenges involved in data interpretation, the continued dependency on the preference of rifampicin as an CYP3A4 inducer needs immediate re-appraisal.

\section{Introduction}

A majority of drugs undergo metabolism via cytochrome P450 (CYP) enzymes, particularly CYP3A4 [1, 2]. Therefore, in early drug development, greater importance has been given to evaluation of the potential for drug-drug interactions (DDIs) due to inhibition and induction of CYP3A4. This aspect is particularly relevant to many anticancer drugs whose metabolic disposition is governed by CYP3A4 [3-8]. Whereas CYP3A4 inhibition may inadvertently increase exposure to the drug beyond the safe therapeutic index, CYP3A4 induction may drastically reduce the exposure, resulting in possible efficacy failure. Ketoconazole and rifampicin are the standard probe substrates used for, respectively, inhibition and induction of 
CYP3A4 in clinical protocols to assess the potential for DDIs.

DDIs with ketoconazole are understood and predictable; however, DDIs with rifampicin have always remained complex because rifampicin is involved to various degrees in the physiological processes that govern absorption, metabolism, distribution (uptake transporters), and excretion (efflux transporters and biliary excretion) of the coadministered drugs [9-19], as depicted in Fig. 1.

Rifampicin is rapidly absorbed in humans, generally within $2 \mathrm{~h}$, with an approximate peak concentration $\left(C_{\max }\right)$ of $10 \mu \mathrm{g} / \mathrm{ml}$ after a standard single oral dose of $600 \mathrm{mg}$ [19]. It has been suggested that higher doses of rifampicin may saturate the metabolism and liver uptake of rifampicin, which may result in a disproportionate increase in rifampicin plasma levels [19]. The main metabolite of rifampicin is formed via a deacetylation process in the liver to form desacetylrifampicin, which has been found to be an active metabolite in the antimicrobial activity of the drug. The elimination half-life of rifampicin is $2.5 \mathrm{~h}$, and the mass balance suggests that rifampicin is excreted almost equally between renal and fecal routes. Upon repeated administration, rifampicin showed lower $C_{\max }$ and area under the concentration-time curve (AUC) values because of auto-induction of its own metabolism via CYP3A4 isozymes [19]. Further, the disposition of rifampicin is governed by sinusoidal efflux transporters for biliary excretion $[13,16,17]$ as well as for the formation of the glucuronide conjugate of the desacetylrifampicin, which is also excreted via biliary mechanisms [19].

As shown in Table 1, the main mechanism for DDIs with rifampicin is via the induction CYP3A4 enzyme, with the exception of cediranib where UGT induction was the likely perpetrator. Although rifampicin is used primarily for its ability to induce CYP3A4, its other effects also affect the overall pharmacokinetics of co-administered drugs. In this report, we cite recently published pharmacokinetic data for anticancer drugs such as cabazitaxel, navitoclax, cabozantinib, cediranib, idelalisib, and romidepsin [3-8] to highlight the dilemma with using rifampicin, which is that the potential for DDIs may be paradoxical in nature and unexpected in some situations. Since polypharmacy is increasingly common, the assimilation and systematic evaluation of such pharmacokinetic interaction data will improve understanding when different mechanisms come into play.

\section{Drug-Drug Interactions (DDIs) with Oral Anticancer Drugs}

Table 1 provides the pharmacokinetic interaction data for navitoclax, cabozantinib, idelalisib, and cediranib when coadministered with rifampicin. In three examples, the mechanism was via the induction of CYP3A4 and in one case it was with the induction of UGT.
Fig. 1 Possible areas for potential clinical drug-drug interaction between rifampicin and the co-administered drugs

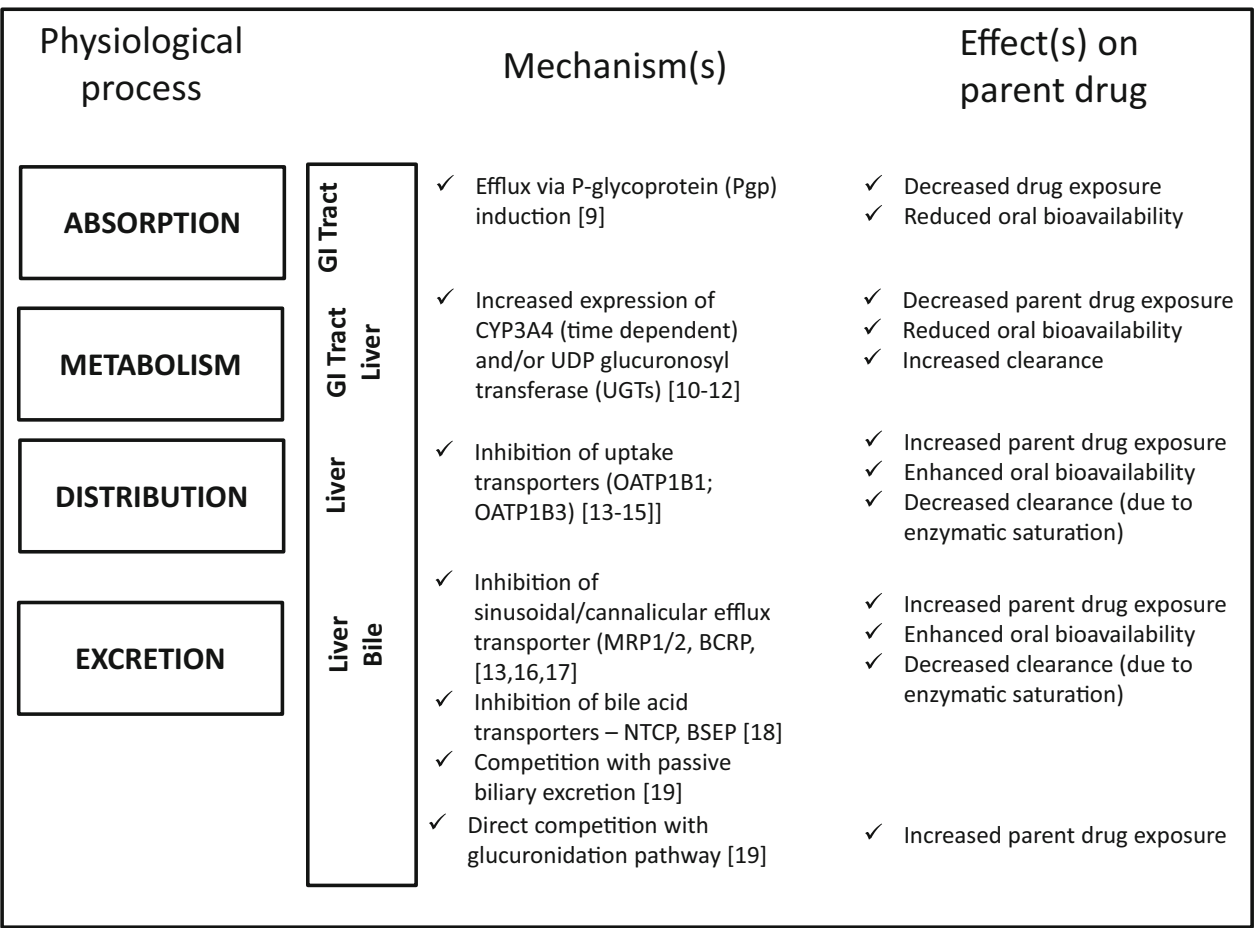




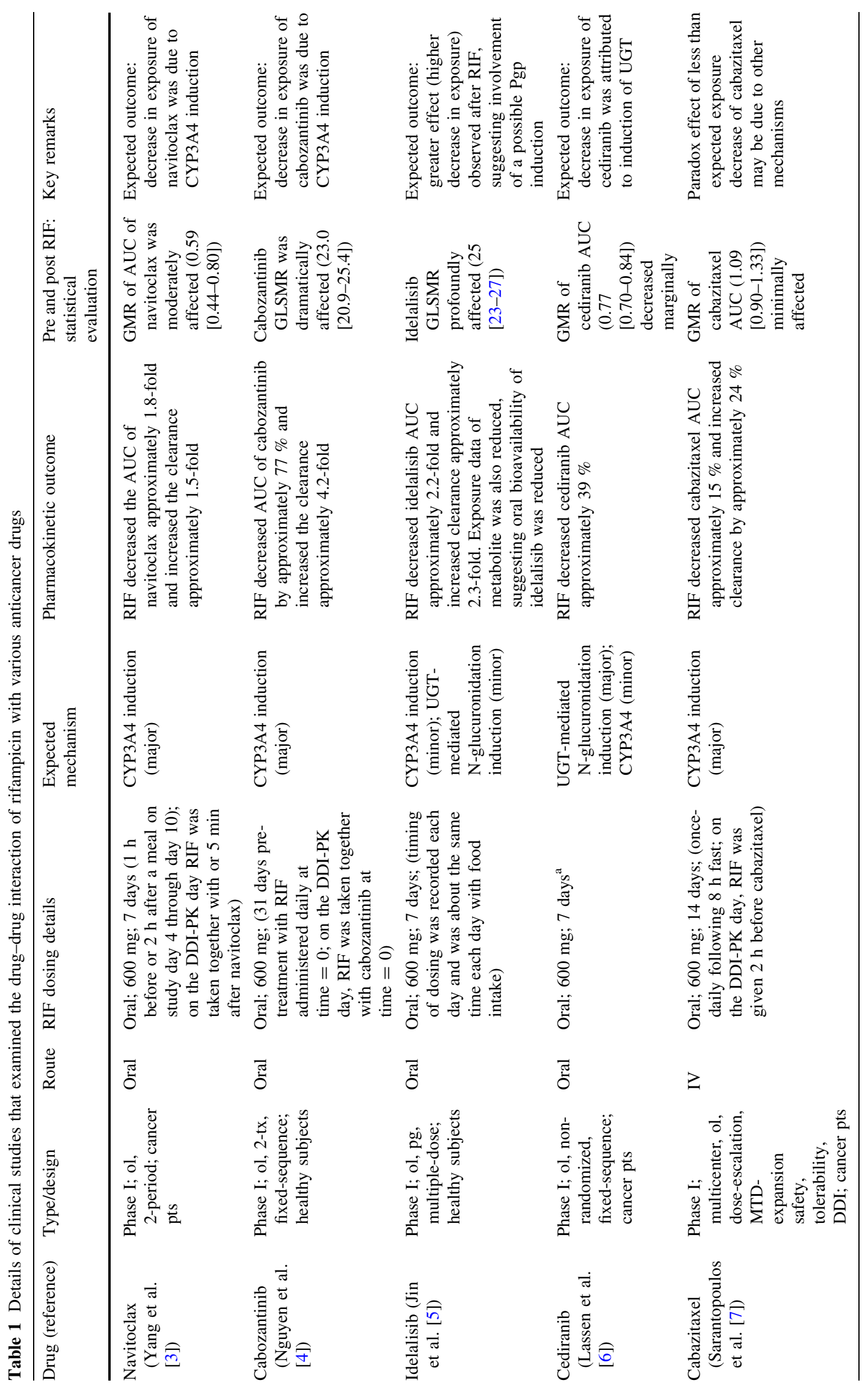




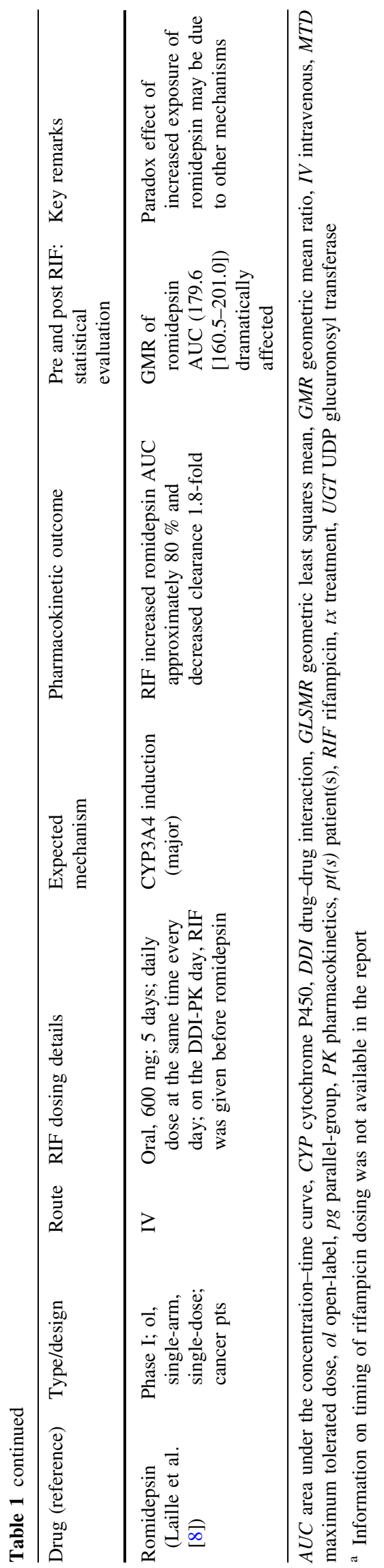

\subsection{Navitoclax}

Navitoclax represents a novel first-in-class small-molecule drug that has a high inhibitory affinity to the Bcl-2 family of receptors [20]. Although the disposition of navitoclax is governed predominantly by hepatic metabolism via CYP3A4 in non-clinical species [3], the human pharmacokinetic data suggested a moderate effect on the clearance of navitoclax when co-administered with rifampicin (Table 1). Earlier data suggested that navitoclax was a substrate for P-glycoprotein (Pgp) [21]. However, since the $C_{\text {max }}$ values of navitoclax were comparable between preand post-rifampicin treatment phases, and rifampicin pretreatment moderately increased the clearance of navitoclax [3], it may be safely concluded that Pgp may possibly play a minor role in the disposition of navitoclax.

\subsection{Cabozantinib}

Cabozantinib is a tyrosine kinase inhibitor with modulations of vascular endothelial growth factor receptor 2 and hepatocyte growth factor receptor [22]. The in vitro metabolism study demonstrated that CYP3A4 was the main CYP enzyme responsible for the N-oxidation of cabozantinib, and inhibition of the CYP3A4 pathway resulted in a $>80 \%$ reduction in the formation of $\mathrm{N}$-oxide metabolite [23]. Given the predominant role of CYP3A4 in the metabolism of cabozantinib [4], pre-treatment with rifampicin showed a profound increase in the clearance of cabozantinib (Table 1). Interestingly, although cabozantinib was also found to be a substrate for several transporters, such as OATP1B1, OATP1B3, and MRP2 [24], rifampicin treatment appeared to have no bearing on these transporters as judged by the clearance of cabozantinib [4]. Additionally, since cabozantinib was also a substrate for Pgp [24], this may also have contributed to the observed higher clearance of the drug during rifampicin treatment [4].

\subsection{Idelalisib}

Idelalisib is a small-molecule drug with high selectivity and affinity for the competitive inhibition of adenosine- $5^{\prime}$ triphosphate binding to the catalytic subunit of the phosphoinositide 3-kinase (PI3K)- $\delta$ enzyme; the inhibition of $\mathrm{PI} 3 \mathrm{~K} \delta$-Akt signaling resulted in reduced proliferation and induction of apoptosis $[25,26]$. The role of CYP3A4 in the oxidative metabolism of idelalisib was minor because aldehyde oxidase primarily catalyzed the formation of the major metabolite GS-563117, which was devoid of pharmacological activity [5]. Interestingly, idelalisib was noted to be an inhibitor of Pgp, OATP1B1, and OATP1B3, whereas GS-563117 was in vitro studies indicated it was a 
time-dependent CYP3A4 inhibitor [5]. The inclusion of probe substrates such as digoxin (Pgp) and rosuvastatin (OATP1B1 and OATP1B3) confirmed the lack of any clinically meaningful pharmacokinetic interaction of idelalisib upon co-administration of the two drugs [5]. The lack of significant change in the midazolam (CYP3A4 probe) pharmacokinetics upon co-administration with idelalisib refuted the notion that GS-563117 was a time-dependent CYP3A4 inhibitor [5]. In combination, the above key observations suggest the interaction of idelalisib with rifampicin would not be influenced by other competing mechanisms, with the exception of CYP3A4. Because CYP3A4 is a minor enzyme responsible for the conversion of idelalisib to GS-563117, it was thought that CYP3A4 induction may lead to a moderate increase in the clearance of idelalisib. However, the pharmacokinetic data suggest a profound effect on the clearance of idelalisib (Table 1). Therefore, it may be speculated that such an observed effect on the clearance of idelalisib may be only substantiated by induction of the UGT1A4 enzyme and/or Pgp. Because the exposure of GS-563117 profoundly decreased [5], it appeared that Pgp induction may have a greater contribution in explaining the lower bioavailability of both idelalisib and GS-563117 when co-administered with rifampicin.

\subsection{Cediranib}

Cediranib is a highly potent inhibitor of all three subtypes of vascular endothelial growth factors, resulting in significant disruption in tumor angiogenesis and growth inhibition [27, 28]. In vitro studies have confirmed the major contributor for the metabolism of cediranib was UGT1A4 and flavin-containing monooxygenase, resulting in the formation of glucuronide metabolite and oxidative metabolite, respectively [29, 30]. The role of CYP enzymes in general appeared to be minor in nature. As expected, pharmacokinetic interaction with rifampicin showed a modest effect on the decreased exposure or increased clearance of cediranib, which was consistent with the induction of UGT1A4 by rifampicin [10-12] (Table 1).

\section{DDIs with Intravenous Anticancer Drugs}

\subsection{Romidepsin}

Romidepsin is a novel drug, the anticancer activity of which is via histone deacetylase (HDAC) inhibitory mechanisms [31, 32]. The major contributor for the extensive hepatic metabolism of romidepsin was CYP3A4, with minor contributions from other CYPs such as 1A1, 2B6, and 2C19 [33]. In a profound paradoxical effect, co- administration of rifampicin increased exposure and decreased clearance of romidepsin by an identical value of approximately 1.8-fold (Table 1). Laille et al. [8] rightly emphasized the complexity involved in DDIs with rifampicin and concluded that inhibition of uptake transporters may have been responsible for the unexpected surge in the plasma exposure of romidepsin in cancer patients. However, Laille et al. [8] opined that, since romidepsin was not a substrate for either OATP1B1 or OATP1B3, the inhibition mediated by rifampicin may possibly occur through inhibition of another as-yet unidentified uptake transporter. Another important mechanism that may possibly contribute to the paradoxical phenomenon of rifampicin involves its effect on the sinusoidal efflux transporter-based biliary excretion process of drugs and/or direct competition with phase II glucuronide conjugation pathway followed by passive biliary excretion (Fig. 1). Although mass balance data for romidepsin has not been established in human subjects, romidepsin has been reported to undergo extensive biliary excretion in rats, with almost $80 \%$ of the total intravenous dose accounted for via the biliary excretory pathway [8]. Hence, it could be reasonably hypothesized that continuous daily oral administration of rifampicin in patients with cancer may have inhibited the MRP-2 transporters, which in turn resulted in a reduced biliary excretion of romidepsin, explaining the high exposure observed in the DDI study with rifampicin [8]. However, since romidepsin does not appear to be a substrate for MRP2 [34], it may be speculated that rifampicin may directly compete with the glucuronidation pathway of romidepsin and/or passive biliary excretion of romidepsin. It should be noted that rifampicin undergoes significant phase II metabolism, and an efficient biliary excretion of the parent drug and metabolites in humans has been observed [19].

\subsection{Cabazitaxel}

Cabazitaxel is a novel taxane agent recently approved for the treatment of hormone-refractory metastatic prostate cancer in combination with either prednisone or prednisolone [32]. The primary metabolic pathway for cabazitaxel was governed by the sole enzyme CYP3A4, contributing to $80-90 \%$ of the total metabolism of the drug [35]. The pharmacokinetic interaction data with rifampicin indicated the impact was marginal at best, suggesting the likelihood of other competing mechanisms outside of CYP3A4 (Table 1). In this context, Ridoux et al. [36] recently published the mass balance, disposition, and excretion data of $\left[{ }^{14} \mathrm{C}\right]$ cabazitaxel in cancer patients. Whereas the renal excretion of cabazitaxel was very limited (approximately $3 \%$ ), the major route of elimination of $\left[{ }^{14} \mathrm{C}\right]$ cabazitaxel was in the feces (approximately $76 \%$ ), presumably due to predominant biliary excretion of the 
parent drug and the metabolites [36]. Because of its CYPrelated metabolism, several oxidative metabolites were formed and detected systemically [36], it is therefore highly likely that phase II metabolism of some of these oxidative metabolites promote biliary excretion process. Therefore, in a situation akin to that of romidepsin, possible inhibition of sinusoidal biliary efflux transporter may have led to higher levels of cabazitaxel. Also, the direct competition of UGT enzymes between phase II metabolite formations for rifampicin versus cabazitaxel may be an additional contributing factor to explaining the higher levels of cabazitaxel, which would counter the loss of drug exposure due to induced CYP3A4 metabolism. In totality, it appears that the reason why a higher drop in cabazitaxel was not observed in this study [7] may be due to factors such as inhibition of biliary efflux transporter and direct completion with UGT enzymes.

\section{Challenges and Perspectives}

The few examples of anticancer drugs discussed in this report demonstrate dilemmas in the interpretation of pharmacokinetic data after administration of rifampicin. The hypothesis for the planned DDI studies of the anticancer drugs with rifampicin was primarily driven by the impact of increased metabolism due to CYP3A4 induction. However, this hypothesis was proven for the oral anticancer agents, although it appeared that in some cases other intrinsic mechanisms, such as Pgp induction and/or UGT induction, may have played an important role alongside the induced CYP3A4 enzymes. Four important observations from the oral anticancer drugs that need careful assessment would be as follows:

1. When CYP3A4 was considered to play a minor role with idelalisib, the greater than expected decrease in exposure to idelalisib [5] was a clear reflection of the important role of the induced Pgp efflux in reducing the oral bioavailability of idelalisib.

2. When CYP3A4 was considered to play a major role with navitoclax based on preclinical work in other species, the observed moderate effect on navitoclax exposure [3] suggested other mechanisms may enable the absorption of navitoclax to escape the likely firstpass effect from the induced CYP3A4. Because navitoclax has a higher long-chain triglyceride solubility, a preferential lymphatic transport may be possible, as observed in a canine study [37].

3. In the case of cediranib, although the CYP3A4 enzyme represented a minor pathway, UGT induction appeared to be key in explaining the reduced cediranib exposure [6].
4. The inhibitory role of rifampicin on uptake transporters (OATP1B1 and OATP1B3) and efflux transporter (MRP2) [13-15] did not appear to adversely affect the clearance of cabozantinib [4], which is an enigma.

Unfortunately, the hypothesis that CYP3A4 induction would decrease CYP3A4 substrate exposure appeared paradoxical for intravenous romidepsin [8] and to a somewhat lesser extent for cabazitaxel [7]. The dilemma with intravenous drugs was that they escape the first-pass metabolism effect of rifampicin as a result induction of both intestinal CYP3A4 and UGT, along with the induced Pgp efflux. Hence, the extent of hepatic CYP3A4 and/or UGT induction may determine the reduced exposure of intravenous drugs. However, other rifampicin mechanisms may counter this phenomenon (Fig. 1). The inhibition of uptake transporters (OATP1B1 and OATP1B3) and biliary sinusoidal efflux transporter (MRP2) by rifampicin [13-15] may render higher exposure of such anticancer drugs, which are substrates to any of the above transporters. While romidepsin was not a substrate for any of them, whether cabazitaxel was a substrate to these transporters is yet to be established. Nevertheless, it appeared that potential areas for DDIs between rifampicin versus romidepsin/cabazitaxel may be due to competitive passive biliary excretion and/or direct competition with UGTs for the phase II glucuronide formation. It should be noted that a high dose of rifampicin was administered for either 14 days (cabazitaxel) or 5 days (romidepsin), and because the UGT system is important for the disposition of rifampicin, UGT depletion over time may become relevant for both cabazitaxel and romidepsin and possibly explain the interaction with rifampicin. In this context, a similar effect—oral drug with profound DDIs with an intravenous drug acting at the biliary excretion level (presumably via a passive biliary excretory pathway and/ or interference at the phase II metabolism) - has been previously demonstrated between cyclosporine and tigecycline in transplant patients with infections [38]. Because tigecycline can efficiently compete at the glucuronidation pathway and with the biliary excretion of cyclosporine, it drastically increased the plasma exposure of the oral cyclosporine, the disposition of which is largely governed by the biliary excretory pathway [38] Other processes may have a lower chance of causing an unexpected outcome in clinical DDI studies with rifampicin: protein-binding displacement, influence on the disposition of the active metabolite(s), and changes to the intestinal microflora may affect the local absorption and metabolism/transporter environment.

Given the observed problems with the interpretation of pharmacokinetic data with rifampicin, several questions 
need to be considered: (1) Should a uniform dosing regimen be considered for DDI studies involving rifampicin (5 days $[n=1], 7$ days $[n=3], 11$ days $[n=1], 14$ days $[n=1])$ ? (2) Does rifampicin serve as the right probe to induce CYP3A4 enzymes for the assessment of pharmacokinetics of CYP3A4 substrate drugs? (3) Should rifampicin be considered a CYP3A4 inducer when intravenous drug pharmacokinetics are assessed for CYP3A drug substrates? (4) Does rifampicin exert similar DDI potential for the same CYP3A4 drug substrate regardless of its route of administration? (5) Aside from the above questions, how should one value the dosing recommendation that arises from the DDI with rifampicin given the complex interplay due to competing mechanisms? (6) Does the dosing recommendation, if any, from rifampicin clinical DDI studies hold good if other CYP3A4 inducers are ingested during the course of the therapy (e.g., St John's Wort)? (7) What should be our level of preparedness with respect to understanding the characteristics of the drug substrate before we undertake a DDI study with rifampicin?

\section{Conclusions}

In summary, paradoxical effects with rifampicin are not new and have been observed in antiretroviral therapy [39]. Aside from CYP3A4 induction, rifampicin may induce and/or inhibit other physiological functions that govern disposition of the drug. Additionally, rifampicin may directly participate in passive excretory processes and indirectly participate in depleting enzymes. The paradoxical effect of rifampicin may differ between oral and intravenous drugs. Furthermore, it is important to understand the types of enzyme(s) and transporter(s) contributing to the clearance of the victim drug prior to using rifampicin as the perpetrator in a planned DDI study, to ensure a reliable prediction on the magnitude of clinical DDI. While the DDI study is planned for an induction phenomenon of CYP3A4 by rifampicin, the repeated rifampicin administration may make it more conducive for a potent transporter inhibition. Therefore, the continued preference for rifampicin as the CYP3A4 inducer needs immediate reappraisal.

\section{Compliance with Ethical Standards}

Conflict of interest NRS has no conflicts of interest that are directly relevant to the content of this paper.

Funding No sources of funding were used to conduct this study or prepare this manuscript.
Open Access This article is distributed under the terms of the Creative Commons Attribution-NonCommercial 4.0 International License (http://creativecommons.org/licenses/by-nc/4.0/), which permits any noncommercial use, distribution, and reproduction in any medium, provided you give appropriate credit to the original author(s) and the source, provide a link to the Creative Commons license, and indicate if changes were made.

\section{References}

1. Rendic S, Di Carlo FJ. Human cytochrome P450 enzymes: a status report summarizing their reactions, substrates, inducers, and inhibitors. Drug Metab Rev. 1997;29:413-580.

2. Sevrioukova IF, Poulos TL. Current approaches for investigating and predicting cytochrome P450 3A4-ligand interactions. Adv Exp Med Biol. 2015;851:83-105.

3. Yang J, Pradhan RS, Rosen LS, Graham AM, Holen KD, Xiong H. Effect of rifampin on the pharmacokinetics, safety and tolerability of navitoclax (ABT-263), a dual inhibitor of Bcl-2 and Bcl-XL, in patients with cancer. J Clin Pharm Ther. 2014;39:680-4.

4. Nguyen L, Holland J, Miles D, Engel C, Benrimoh N, O'Reilly T, et al. Pharmacokinetic (PK) drug interaction studies of cabozantinib: effect of CYP3A inducer rifampin and inhibitor ketoconazole on cabozantinib plasma $\mathrm{PK}$ and effect of cabozantinib on CYP2C8 probe substrate rosiglitazone plasma PK. J Clin Pharmacol. 2015;55:1012-23.

5. Jin F, Robeson M, Zhou H, Moyer C, Wilbert S, Murray B, et al. Clinical drug interaction profile of idelalisib in healthy subjects. J Clin Pharmacol. 2015;55:909-19.

6. Lassen U, Miller WH, Hotte S, Evans TR, Kollmansberger C, Adamson D, et al. Phase I evaluation of the effects of ketoconazole and rifampicin on cediranib pharmacokinetics in patients with solid tumours. Cancer Chemother Pharmacol. 2013;71:543-9.

7. Sarantopoulos J, Mita AC, Wade JL, Morris JC, Rixe O, Mita MM, et al. Phase I study of cabazitaxel plus cisplatin in patients with advanced solid tumors: study to evaluate the impact of cytochrome P450 3A inhibitors (aprepitant, ketoconazole) or inducers (rifampin) on the pharmacokinetics of cabazitaxel. Cancer Chemother Pharmacol. 2014;74:1113-24.

8. Laille E, Patel M, Jones SF, Burris HA 3rd, Infante J, Lemech C, et al. Evaluation of CYP3A-mediated drug-drug interactions with romidepsin in patients with advanced cancer. J Clin Pharmacol. 2015;55:1378-85.

9. Schuetz EG, Schinkel AH, Relling MV, Schuetz JD. P-glycoprotein: a major determinant of rifampicin-inducible expression of cytochrome P4503A in mice and humans. Proc Natl Acad Sci USA. 1996;93:4001-5.

10. Zilly W, Breimer DD, Richter E. Induction of drug metabolism in man after rifampicin treatment measured by increased hexobarbital and tolbutamide clearance. Eur J Clin Pharmacol. 1975;19(9):219-27.

11. Kolars JC, Schmiedlin-Ren P, Schuetz JD, Fang C, Watkins PB. Identification of rifampin-inducible P450IIIA4 (CYP3A4) in human small bowel enterocytes. J Clin Invest. 1992;90:1871-8.

12. Lee SY, Lee JY, Kim YM, Kim SK, Oh SJ. Expression of hepatic cytochrome P450 s and UDP-glucuronosyltransferases in PXR and CAR double humanized mice treated with rifampicin. Toxicol Lett. 2015;235:107-15.

13. Neyt S, Huisman MT, Vanhove C, De Man H, Vliegen M, Moerman $\mathrm{L}$, et al. In vivo visualization and quantification of (Disturbed) Oatp-mediated hepatic uptake and Mrp2-mediated 
biliary excretion of $99 \mathrm{mTc}$-mebrofenin in mice. J Nucl Med. 2013;54:624-30.

14. Anderson MS, Cote J, Liu Y, Stypinski D, Auger P, Hohnstein A, et al. Effects of Rifampin, a potent inducer of drug-metabolizing enzymes and an inhibitor of OATP1B1/3 transport, on the single dose pharmacokinetics of anacetrapib. J Clin Pharmacol. 2013;53:746-52.

15. Khurana V, Minocha M, Pal D, Mitra AK. Inhibition of OATP1B1 and OATP-1B3 by tyrosine kinase inhibitors. Drug Metabol Drug Interact. 2014;29:249-59.

16. Te Brake LH, Russel FG, van den Heuvel JJ, de Knegt GJ, de Steenwinkel JE, Burger DM, Aarnoutse RE, Koenderink JB. Inhibitory potential of tuberculosis drugs on ATP-binding cassette drug transporters. Tuberculosis (Edinb). 2016;96:150-7.

17. Guo YX, Xu XF, Zhang QZ, Li C, Deng Y, Jiang P, et al. The inhibition of hepatic bile acids transporters Ntcp and Bsep is involved in the pathogenesis of isoniazid/rifampicin-induced hepatotoxicity. Toxicol Mech Methods. 2015;25:382-7.

18. Lengyel G, Veres Z, Tugyi R, Vereczkey L, Molnár T, Glavinas $\mathrm{H}$, et al. Modulation of sinusoidal and cannalicular elimination of bilirubin-glucoronides by rifampicin and other cholestatic drugs in a sandwich culture of rat hepatocytes. Hepatol Res. 2008;38:300-9.

19. Acocella G. Clinical pharmacokinetics of rifampicin. Clin Pharmacokinet. 1978;3:108-27.

20. Wilson WH, O'Connor OA, Czuczman MS, LaCasce AS, Gerecitano JF, Leonard JP, et al. Navitoclax, a targeted high-affinity inhibitor of BCL-2, in lymphoid malignancies: a phase 1 doseescalation study of safety, pharmacokinetics, pharmacodynamics, and antitumour activity. Lancet Oncol. 2010;11:1149-59.

21. Vogler M, Dickens D, Dyer MJ, Owen A, Pirmohamed M, Cohen GM. The B-cell lymphoma 2 (BCL2)-inhibitors, ABT-737 and ABT-263, are substrates for P-glycoprotein. Biochem Biophys Res Commun. 2011;408:344-9.

22. Yakes FM, Chen J, Tan J, Yamaguchi K, Shi Y, Yu P, et al. Cabozantinib (XL184), a novel MET and VEGFR2 inhibitor, simultaneously suppresses metastasis, angiogenesis, and tumor growth. Mol Cancer Ther. 2011;10:2298-308.

23. COMETRIQ ${ }^{\mathrm{TM}}$ (cabozantinib) capsules. US Prescribing Information. USA: Exelixis, Inc.,; 2012.

24. Lacy S, Hsu B, Miles D, Aftab D, Wang R, Nguyen L. Metabolism and disposition of cabozantinib in healthy male volunteers and pharmacologic characterization of its major metabolites. Drug Metab Dispos. 2015;43:1190-207.

25. Lannutti BJ, Meadows SA, Herman SE, Kashishian A, Steiner B, Johnson AJ, et al. CAL-101, a p110delta selective phosphatidylinositol-3-kinase inhibitor for the treatment of B-cell malignancies, inhibits PI3K signaling and cellular viability. Blood. 2011;117:591-4.

26. Hoellenriegel J, Meadows SA, Sivina M, Wierda WG, Kantarjian $\mathrm{H}$, Keating MJ, et al. The phosphoinositide 30-kinase delta inhibitor, CAL-101, inhibits B-cell receptor signaling and chemokine networks in chronic lymphocytic leukemia. Blood. 2011;118:3603-12.

27. Heckman CA, Holopainen T, Wirzenius M, Keskitalo S, Jeltsch M, Yla-Herttuala S, et al. The tyrosine kinase inhibitor cediranib blocks ligandinduced vascular endothelial growth factor receptor3 activity and lymphangiogenesis. Cancer Res. 2008;68:4754-62.

28. Wedge SR, Kendrew J, Hennequin LF, Valentine PJ, Barry ST, Brave SR, et al. AZD2171: a highly potent, orally bioavailable, vascular endothelial growth factor receptor-2 tyrosine kinase inhibitor for the treatment of cancer. Cancer Res. 2005;65:4389-400.

29. Lenz EM, Spear M, Drake C, Pollard CR, Ward M, SchulzUtermoehl $\mathrm{T}$, et al. Characterisation and identification of the human $\mathrm{N}$-glucuronide metabolite of cediranib. J Pharm Biomed Anal. 2010;53:526-36.

30. Schulz-Utermoehl T, Spear M, Pollard CR, Pattison C, Rollison $\mathrm{H}$, Sarda $\mathrm{S}$, et al. In vitro hepatic metabolism of cediranib, a potent vascular endothelial growth factor tyrosine kinase inhibitor: interspecies comparison and human enzymology. Drug Metab Dispos. 2010;38:1688-97.

31. Kim M, Thompson LA, Wenger SD, O’Bryant CL. Romidepsin: a histone deacetylase inhibitor for refractory cutaneous T-cell lymphoma. Ann Pharmacother. 2012;46:1340-8.

32. Coiffier B, Federico M, Caballero D, Dearden C, Morschhauser $\mathrm{F}$, Jäger $\mathrm{U}$, et al. Therapeutic options in relapsed or refractory peripheral T-cell lymphoma. Cancer Treat Rev. 2014;40:1080-8.

33. Shiraga T, Tozuka Z, Ishimura R, Kawamura A, Kagayama A. Identification of cytochrome $\mathrm{P} 450$ enzymes involved in the metabolism of FK228, a potent histone deacetylase inhibitor, in human liver microsomes. Biol Pharm Bull. 2005;28:124-9.

34. ISTODAX ${ }^{\circledR}$ (romidepsin). Prescribing information. http://www. istodax.com/pdfs/ISTODAX_PackageInsert.pdf. Accessed 16 Nov 2015.

35. Sanofi U.S. LLC. JEVTANA ${ }^{\circledR}$ (cabazitaxel) Injection, Prescribing Information. Bridgewater, NJ, USA, 2010. Available at: http://www.accessdata.fda.gov/drugsatfda_docs/label/2010/2010 23lbl.pdf.

36. Ridoux L, Sémiond DR, Vincent C, Fontaine H, Mauriac C, Sanderink GJ, et al. A phase I open-label study investigating the disposition of [14C]-cabazitaxel in patients with advanced solid tumors. Anticancer Drugs. 2015;26:350-8.

37. Choo EF, Boggs J, Zhu C, Lubach JW, Catron ND, Jenkins G, et al. The role of lymphatic transport on the systemic bioavailability of the Bcl-2 protein family inhibitors navitoclax(ABT263) and ABT-199. Drug Metab Dispos. 2014;42:207-12.

38. Srinivas NR. Tigecycline and cyclosporine interaction-an interesting case of biliary-excreted drug enhancing the oral bioavailability of cyclosporine. Eur J Clin Pharmacol. 2009;65:543-4.

39. Srinivas NR. Unsuspected and paradoxical potential for drug interaction by rifampin: things to ponder with antiretroviral therapy. J Infect Dis. 2009;199:766-7. 